\title{
Organization and evolution of the polyphenol oxidase gene family in barley
}

\author{
Levanova N.M. ${ }^{1,2}{ }^{*}$, Glagoleva A.Y. ${ }^{1}$, Khlestkina E.K. ${ }^{1,2,3}$, Shoeva O.Y. ${ }^{1}$ \\ ${ }^{1}$ Institute of Cytology and Genetics, SB RAS, Novosibirsk, Russia \\ ${ }^{2}$ Novosibirsk State University, Novosibirsk, Russia \\ ${ }^{3}$ N.I. Vavilov All-Russian Institute of Plant Genetic Resources, St. Petersburg, Russia \\ *e-mail:n.levanova@g.nsu.ru
}

Key words: Hordeum vulgare, Ppo, polyphenol oxidase, salt stress, pigmentation

Motivation and Aim: Tyrosinases are ubiquitous enzymes among all living organisms. In plants, tyrosinases called polyphenol oxidases (PPO) are involved in the response to abiotic and biotic stresses. In cereals, Ppo is predominantly represented by a gene family. Two Ppo genes were previously identified in barley: Ppol and Ppo2, but additional copies were expected. The aim of the current study was to characterize the Ppo gene family in barley, to analyze the functional activity of the Ppo genes and their evolutionary relationships with orthologs of other cereals.

Methods and Algorithms: We used the NCBI database, IPK Barley BLAST Server and EnsemblPlants for sequences identification. For sequence alignment and the evolutionary tree construction, MULTALIN and MEGA 7.0 software were used. Cis-regulatory elements were analyzed using PLACE database. We developed specific primers for each copy using PrimerQuestTool. We used RT-PCR and qPCR to analyze expression of the Ppo genes.

Results: We identified two additional genes Ppo3 and Ppo4 based on the previously reported Ppo1 and Ppo 2 and located them on chromosomes $3 \mathrm{H}$ and $4 \mathrm{H}$, respectively. Copies contain the conservative tyrosinase domain, however, have a different intronexon structure, as well as predicted promoter structure. Expression analysis in various organs (root, coleoptile, leaf, stem, developing spike, pericarp, and hulls) and also under salt stress showed that the Ppo genes possess different expression patterns. At the constructed evolutionary tree $P p o 1$ and $P p o 2$ clustered separately from copies of Ppo3 and Ppo4. Presumably, the formation of the gene cluster comprising the Ppol and $P$ po 2 genes occurred as a result of segment duplication in the common ancestor of the Triticeae tribe. Orthologs of the Ppo3 and Ppo4 genes of barley were detected in other cereal species.

Conclusion: The Ppo gene family in barley contains at least four genes that maintain their functional activity during evolution.

Acknowledgements: Supported by the Russian Science Foundation (No. 16-14-00086). 